\title{
Implementación de la Gestión de Riesgo en el proyecto MacroFacultad de Ingeniería-Chile
}

\author{
Peter Backhouse E., Doctor ${ }^{1}$, Leticia Galleguillos P., Doctor $(c)^{1}$, and Camila Zapata C., Licenciada ${ }^{1}$ \\ ${ }^{1}$ Universidad del Bío-Bío, Chile,pbackhou@ubiobio.cl,.lgalleguillos@ubiobio.cl,czapata@ubiobio.cl
}

\begin{abstract}
El análisis de riesgos realizado para el proyecto "World Class Engineering at Regional State Universities in the Central-South of Chile" (Ingeniería de Clase Mundial en las Universidades estatales regionales de la Zona Centro-Sur de Chile), se realizó considerando las indicaciones establecidas en la norma ISO 31.000 y teniendo como antecedentes todos los documentos evaluados durante las etapas consecutivas del proyecto, los reglamentos institucionales y las opiniones de las personas que han trabajado activamente en el proyecto durante estos ya casi tres años desde los inicios de la etapa de formulación.
\end{abstract}

Este análisis dio origen a una cantidad no menor de posibles potenciales riesgos que podrían ocurrir en el avance de proyecto, tanto en ámbitos estratégicos como operativos, tal como se describirá más adelante, lo cual refleja el espíritu crítico y realista que ha existido al evaluar el funcionamiento del equipo de trabajo y sus resultados.

Las principales consideraciones de este análisis tomaron las sugerencias derivadas de la aplicación de los estándares de la ISO 31000 y los requerimientos adicionales, entre los cuales estaba en primera instancia la definición de los Principios y el Marco de Trabajo para la gestión de riesgos del proyecto, lo que implicó la definición del proceso de gestión de riesgos a usar, el que incluyó el establecimiento del contexto, la valoración de los riesgos y el tratamiento de los mismos. Por su parte, este procedimiento debía incluir una definición del contexto del proyecto, el establecimiento de objetivos, la identificación de riesgos, la respuesta a los riesgos (medidas de mitigación para todos los riesgos, planes de contingencia para riesgos mayores), y el monitoreo y comunicación (reportes e informes). Además, todos estos aspectos se reflejaron en la denominada "Matriz de Riesgos", la cual incluía la descripción y clasificación de los riesgos estratégicos y operacionales, el nivel de severidad de cada riesgo (alto, medio y moderado) y su probabilidad de ocurrencia, la tipología de cada riesgo, las medidas de mitigación aplicables y las métricas para su monitoreo (Key Risk Indicators, KRIs).

El otro aspecto relevante, correspondió a los criterios para la Valoración de los Riesgos, para lo cual se utilizaron los niveles alto, medio y bajo para la probabilidad de ocurrencia del riesgo y alta, media y baja para la consecuencia del riesgo, estableciéndose un rango de probabilidades y consecuencias, desde la probabilidad alta, que implica que es casi seguro que el evento ocurrirá, 1 o más veces, durante el proyecto, hasta la probabilidad baja, la cual implica que es improbable que el evento ocurra durante el proyecto. Similar descripción se utilizó con las consecuencias, teniéndose el rango entre la consecuencia alta, que implica que el riesgo impide que se logre el objetivo definido y provoca daño significativo al proyecto, hasta la consecuencia baja, en la cual, el riesgo tiene un efecto menor en la consecución del objetivo, que no afecta significativamente los atributos definidos.

Por último, al hacer el análisis detallado para determinar cuáles son los riesgos a los que la MacroFacultad debe ponerle mayor atención, se puede desprender que, considerando aquellos riesgos cuyos resultados globales (Probabilidad de ocurrencia $x$

Digital Object Identifier (DOI): http://dx.doi.org/10.18687/LACCEI2016.1.1.087 ISBN: 978-0-9822896-9-3

ISSN: $2414-6390$
Consecuencias) son más elevados, se identifican los riesgos asociados al "incumplimiento de los hitos críticos esperados en los tiempos planificados", la "dedicación insuficiente por parte del cuerpo académico de las Facultades, al proyecto", el "tiempo de demora en el cumplimiento de objetivos, por motivos de burocracia interna universitaria”, el "desbalance en la Vinculación con el Medio de cada una de las Universidades", la "calidad de proveedores por debajo de los estándares esperados", las "diferencias entre las definiciones de CORFO y del Ministerio de Educación, que limitan las acciones del equipo de trabajo”, "vinculación con el medio externo se ve afectado por la reforma educacional, lo que limita las acciones del equipo de trabajo" y por último el riesgo asociado a que los "segmento de interés definido por la MacroFacultad no están interesado en las actividades desarrolladas por el Proyecto".

Keywords-Riesgo, ISO 31000, Matriz de Riesgos, Probabilidad, Consecuencias, Indicadores Claves.

\section{MARCO CONCEPTUAL: GESTIÓN DE RIESGO}

El Risk Managament es un mecanismo que ayuda a predecir y manejar eventos que puedan evitar que el proyecto culmine antes de lo estipulado (Amendola, 2007), y que tiene como principal clave el seguir una secuencia de procesos interrelacionados (Medir, Monitorear y Mitigar los riesgos) como precisa Roisenzvit \& Zárate (2006).

En primer lugar, se entenderá como riesgo a "La posibilidad de que un evento ocurra $y$ afecte desfavorablemente al logro de objetivos de una organización" (Committee of Sponsoring Organizations of the Treadway Commission, 2004), además, se debe considerar que la incertidumbre o el riesgo será inherente a todas las actividades de una empresa u organización (International Organization for Standardization, 2009), y es ahí donde radica la importancia de la correcta definición de los riesgos dentro de cualquier entidad, ya que le podrá otorgar una base sólida a la gestión de dicha organización.

Los riesgos son factores que pueden afectar el cumplimiento de los objetivos, pudiendo ser internos o externos, los cuales cambian a lo largo del tiempo, por tanto, lo que hoy es riesgo, mañana puede no serlo y viceversa. La Gestión de riesgo implicará realizar un análisis de este, el cual deberá considerar dos puntos importantes: un estudio de la factibilidad de que ocurra dicho evento, es decir, la probabilidad; Y el impacto que tendría si realmente ocurriese, o sea, el impacto (Amendola, 2007).

Según explica Kevin W. Knight, jefe del trabajo ISO que desarrolló la norma ISO 31000:2009, “Todas las organizaciones, sin importar que tan grande o pequeñas, se enfrentan a factores tanto internos como externos que crean

$14^{\text {th }}$ LACCEI International Multi-Conference for Engineering, Education, and Technology: "Engineering Innovations for Global Sustainability", 20-22 July 2016, San José, Costa Rica. 
incertidumbre sobre si estas podrán alcanzar sus objetivos. El efecto de esta incertidumbre es "riesgo" y es inherente en todas sus actividades." (International Organization for Standardization, 2009).

En particular, como primer paso para recopilar información de lo que involucraría un Sistema de Gestión de Riesgos (SGR) dentro de la MacroFacultad, se recurrió a la literatura de dos sistemas o normas de control:

- ISO 31000 "Gestión del Riesgo, principios y directrices": Norma que describe la Gestión del riesgo, sus principios y orientaciones. No está prevista para fines de certificación, su objetivo es el de constituirse en una base y guía para las empresas que decidan implementar la gestión de riesgo. Se establecen principios, un marco de trabajo y procesos integrados a la cultura de la organización.

- Modelo COSO Enterprise Risk Management II (COSO ERM II): COSO solicita a la empresa de auditoria Price Waterhouse Coopers (PwC), desarrollar un marco que fuera accesible para el uso de la dirección de las empresas y ayudar en la mejora de la gestión de riesgos en las organizaciones, el COSO ERM II, este pretende ser una guía actualizada de herramientas para ayudar a las empresas en la administración de sus riesgos de una forma más global, con un énfasis aún mayor en lo que es el control interno.

Si bien son dos modelos que abordan un mismo tema, la forma en que lo describen y trabajan es diferente. Cada organización es libre de decidir cuál de estos modelos se puede adaptar mejor a sus necesidades, y debido a que no se consideran alternativas excluyentes, pueden incluso ser complementadas y lograr captar lo mejor de cada una de ellas (González, 2013).

El principal resultado de este sistema de gestión es llegar a definir la Matriz de riesgo. Como se indica en García (2007), la matriz de riesgo, o matriz de probabilidad e impacto, es una herramienta que permite clasificar e identificar gráficamente el nivel de riesgo que tiene una determinada actividad, y por ende conocer cuál de ellos puede requerir una atención prioritaria. Con los riesgos ya identificados, se debe determinar cuál es la probabilidad de ocurrencia y nivel de impacto de cada uno de los eventos (García, 2007), esto permitirá construir una matriz en base a escalas cualitativas en ambos criterios.

\section{IMPLEMENTACIÓN DE LA GESTIÓN DE RIESGOS EN LA MACROFACULTAD}

En base a lo anterior, y posterior a la revisión de la literatura existente sobre Risk Managament, el procedimiento para la construcción e implementación de un SGR se lleva a cabo según la siguiente metodología de trabajo:

1. Levantamiento de información: esto se realizó a través de entrevistas al equipo de trabajo, análisis de documentación y encuestas. Luego de comprender el procedimiento asociado al análisis de riesgos, se realizó la etapa de recolección de información primaria, complementada con una revisión exhaustiva de todos los documentos generados en el marco del presente proyecto, más los documentos que reglamentan el funcionamiento de las tres instituciones, de manera de tener claro todos los aspectos que puedan influir en el logro de los objetivos planteados por la Macro Facultad. Como ya se dijo, esta revisión, se complementó con un levantamiento de información a través de entrevistas y encuestas a los participantes e involucrados en el proyectos, de manera de generar un listado detallado de potenciales riesgos que puedan afectar el correcto desarrollo de Proyecto. Estas actividades se reflejan en la Tabla 1.

\begin{tabular}{|l|c|}
\hline Entrevistas individuales y grupales & 12 \\
\hline Entrevistas telefónicas & 3 \\
\hline Análisis de Documentos & 6 \\
\hline Encuestas online & 12 \\
\hline
\end{tabular}

Tabla 1. Descripción de Instrumentos utilizados para la recolección de información.

Fuente: Elaboración propia

2. Tipos de riesgo: A través de la metodología del Project Managament Institute PMI, la cual enumera las categorías y subcategorías de donde pueden surgir riesgos para un proyecto. Un beneficio de este enfoque es que recuerda a los participantes de un ejercicio de identificación de riesgos, las múltiples fuentes de donde pueden surgir riesgos en el proyecto. Esto se adapta a los requerimientos solicitados por CORFO, con lo que se obtienen las divisiones mostradas en la Figura $\mathrm{N}^{\circ} 1$. A esta división categorías, se suma "Ejecución", la cual apunta a las actividades directamente relacionadas al desarrollo del proyecto.

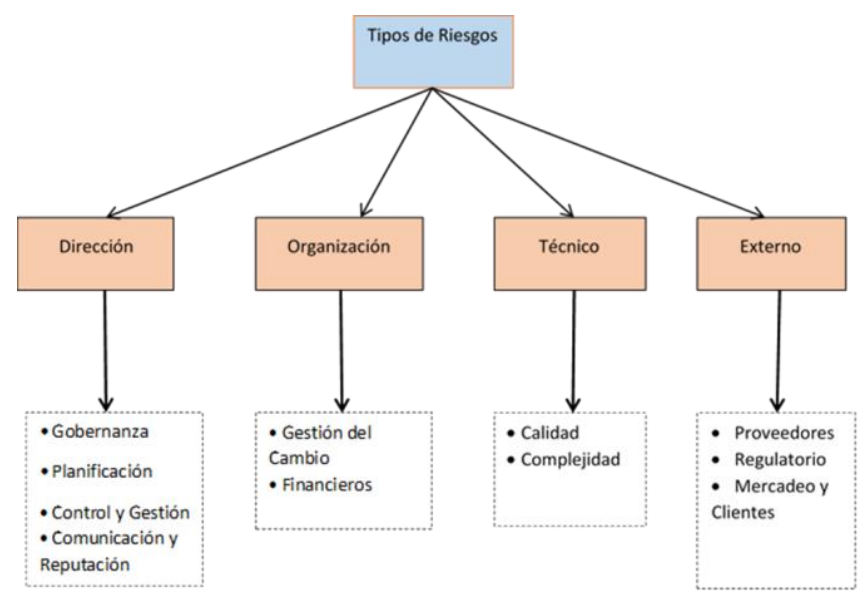

Fig. 1. Tipos de riesgos involucrados Fuente: Elaboración propia 
3. Análisis y tratamiento de los riesgos: Se elaboró una matriz de riesgo de $3 \times 3$ como se muestra en la Figura $\mathrm{N}^{\circ}$ 2, considerando los niveles de riesgo: Moderado, Medio y Alto.

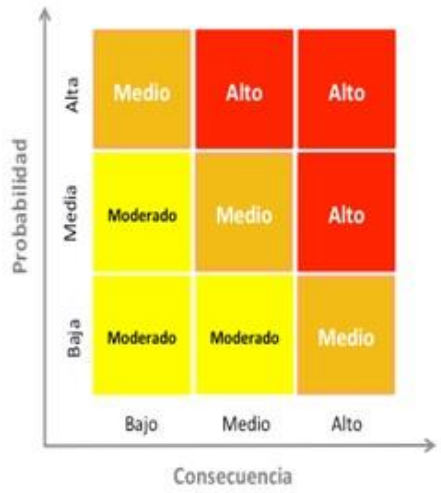

Fig. 2. Matriz de riesgo

Fuente: Elaboración propia

Por tanto, a cada riesgo se le determinó la probabilidad que ocurrencia, y el nivel de impacto que tendría si se llega a dar el evento, con esto se obtiene el nivel de riesgo final para cada uno de ellos. Es importante resaltar, que tal como se muestra en la Tabla 2, para mayor claridad, se utilizó un esquema de colores para especificar el rango de valores, considerando el rojo el valor más alto o riesgosos, el anaranjado, para los valores intermedios, y el color amarillo para los valores bajos. El análisis y tratamientos de los riesgos, se realizó considerando las probabilidades de ocurrencia de cada uno de los riesgos detectados después de realizar el análisis de la información recopilada, y sus correspondientes consecuencias, cada una de ellas con una escala de tres valores: Baja, Media, Alta, lo que generó la tabla de posibles valores mostrada a continuación:

Riesgo $=$ Probabilidad $\times$ Consecuencia

\begin{tabular}{|c|c|c|}
\hline P & C & R \\
\hline Probabilidad & Consecuencia & Riesgo \\
\hline Baja & Baja & Moderado \\
\hline Baja & Media & Moderado \\
\hline Baja & Alta & Medio \\
\hline Media & Baja & Moderado \\
\hline Media & Media & Medio \\
\hline Media & Alta & Alto \\
\hline Alta & Baja & Medio \\
\hline Alta & Media & Alto \\
\hline Alta & Alta & Alto \\
\hline
\end{tabular}

Tabla 2. Descripción de posibles valores asignados a cada riesgo. Fuente: Elaboración propia
Además, para cada riesgo, se caracterizó la descripción del tratamiento que tendrá cada uno de ellos, el riesgo residual que existiría, a través de qué herramientas o cómo se hará el monitoreo y con qué periodicidad se medirá, por último, se definen los KRIs (Key Risk Indicators o Indicadores Claves de Riesgo) con su máximo nivel aceptable y fórmula de cálculo.

Los KRI contribuyen a la toma de decisiones a través del establecimiento de umbrales mínimos y rangos de tolerancia para los diversos riesgos, los que deberían ser definidos por los directivos de la organización. Es recomendable realizar un seguimiento del nivel de riesgo, revisando los KRI en forma periódica y sistemática para alertar cambios que puedan indicar problemas.

A partir de lo anterior, y según la metodología de trabajo expuesta, se logran identificar los riesgos asociados tanto a la formulación como al desarrollo del proyecto, a nivel de organización y operación, con esto se pretende abordar un amplio espectro de eventos que pueden presentarse y no resultar según lo planificado.

Cabe mencionar, que, si bien se puede pensar que un SGR tiene una mirada negativa de la organización, debido a que destaca aquellos elementos que pueden resultar mal, esto implica que es más bien una visión realista de lo que puede acontecer, y el principal objetivo es que la organización tenga conciencia de todos los riesgos que conllevan sus propias operaciones y se transforme en un mapa de cómo proceder si algo no resulta según lo contemplado.

En el caso particular de la MacroFacultad, se definieron un total de 64 riesgos, y para cada uno de éstos, se asocian todos los elementos descritos con anterioridad, además de su clasificación en niveles de riesgo, generándose una Matriz de Riesgos bastante extensa, la cual contiene los siguientes campos:

- Riesgo

- Descripción del Tratamiento

- Riesgo Residual

- Monitoreo

- Periodicidad de Monitoreo

- Descripción del Monitoreo

- Indicadores de Riesgo Claves (KRIs)

- Valor Máximo aceptable KRI

- Responsable

- Estado Inicial

- Monitoreo 1

- Monitoreo 2

- Monitoreo N

A modo de ejemplo, en la tabla $\mathrm{N}^{\circ} 3$ se presentan aquellos riesgos que, según lo analizado por el equipo de trabajo, se clasificaron en un nivel alto de riesgo, y por tanto deberían tener una atención prioritaria.

14 ${ }^{\text {th }}$ LACCEI International Multi-Conference for Engineering, Education, and Technology: "Engineering Innovations for 


\begin{tabular}{|c|c|c|c|}
\hline $\begin{array}{l}\text { Tipo de } \\
\text { Riesgo } \\
\end{array}$ & Riesgo & $\begin{array}{c}\text { Descripción del } \\
\text { Tratamiento }\end{array}$ & $\begin{array}{c}\text { Riesgo } \\
\text { Residual } \\
\end{array}$ \\
\hline Dirección & $\begin{array}{l}\text { Incumplimien } \\
\text { to de los } \\
\text { hitos críticos } \\
\text { esperados en } \\
\text { los tiempos } \\
\text { planificados. }\end{array}$ & $\begin{array}{lr}\text { Reuniones } & \text { de } \\
\text { monitoreo } & \text { y } \\
\text { evaluación del estado } \\
\text { de avance de las } \\
\text { actividades reridos. }\end{array}$ & $\begin{array}{l}\text { No lograr } \\
\text { cumplimiento, } \\
\text { generando daño } \\
\text { a la imagen, } \\
\text { reputación y } \\
\text { credibilidad de } \\
\text { las } \\
\text { instituciones). } \\
\end{array}$ \\
\hline Organización & $\begin{array}{l}\text { Dedicación } \\
\text { insuficiente } \\
\text { por parte del } \\
\text { cuerpo } \\
\text { académico } \\
\text { de las } \\
\text { Facultades, } \\
\text { al proyecto. } \\
\end{array}$ & $\begin{array}{l}\text { Ver manejo de Riesgo } \\
\text { Recursos: "Equipo de } \\
\text { trabajo carece de } \\
\text { incentivos/tiempo para } \\
\text { desarrollar } \\
\text { proyecto". }\end{array}$ & $\begin{array}{l}\text { No lograr } \\
\text { calidad } \\
\text { esperada en } \\
\text { actividades } \\
\text { desarrolladas } \\
\text { (ver manejo de } \\
\text { este riesgo en } \\
\text { Calidad). } \\
\end{array}$ \\
\hline Organización & $\begin{array}{l}\text { Tiempo de } \\
\text { demora en el } \\
\text { cumplimient } \\
\text { o de } \\
\text { objetivos con } \\
\text { motivo de } \\
\text { burocracia } \\
\text { interna } \\
\text { universitaria. }\end{array}$ & $\begin{array}{l}\text { Establecer prioridad } \\
\text { interna al Proyecto } \\
2030 \text { respaldado por } \\
\text { autoridades } \\
\text { universitarias, por } \\
\text { medio de cartas de } \\
\text { respaldo. }\end{array}$ & $\begin{array}{l}\text { Excesivo control } \\
\text { de autoridades } \\
\text { internas y de } \\
\text { cada contraloría } \\
\text { regional. }\end{array}$ \\
\hline Técnico & $\begin{array}{l}\text { Desbalance } \\
\text { en } \\
\text { Vinculación } \\
\text { con el Medio } \\
\text { de cada } \\
\text { Universidad. }\end{array}$ & $\begin{array}{lr}\text { Primera } & \text { Etapa: } \\
\text { Contratar un informe } \\
\text { externo que evalúe a } \\
\text { cada r Facultad } \\
\text { participanter para } \\
\text { generar r una } \\
\text { evaluación individual } \\
\text { y consolidado que } \\
\text { identifique brechas y } \\
\text { genere un plan de } \\
\text { trabajo conjunto y por } \\
\text { Universidad. } \\
\text { Segundaraple Etapa: } \\
\text { establecer un sistema } \\
\text { de evaluación y de } \\
\text { control de desbalances } \\
\text { y calidad. }\end{array}$ & $\begin{array}{l}\text { No lograr } \\
\text { resultados con } \\
\text { la calidad } \\
\text { esperada. }\end{array}$ \\
\hline Externo & $\begin{array}{l}\text { Calidad de } \\
\text { proveedores } \\
\text { por debajo } \\
\text { de los } \\
\text { estándares } \\
\text { esperados. }\end{array}$ & $\begin{array}{l}\text { Se debe contar con al } \\
\text { menos una terna de } \\
\text { proveedores, de } \\
\text { manera de elegir } \\
\text { calidad y tener poder } \\
\text { de negociación en } \\
\text { precios. }\end{array}$ & $\begin{array}{lr}\text { Ver riesgo en } \\
\text { Calidad } \\
\text { lograr calidad } \\
\text { esperada en } \\
\text { actividades } \\
\text { desarrolladas", }\end{array}$ \\
\hline Externo & $\begin{array}{l}\text { Diferencias } \\
\text { entre } \\
\text { definiciones } \\
\text { de CORFO y } \\
\text { MINEDUC } \\
\text { limitan las } \\
\text { acciones del } \\
\text { equipo de } \\
\text { trabajo. }\end{array}$ & 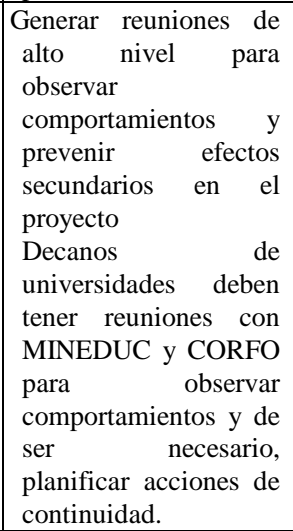 & 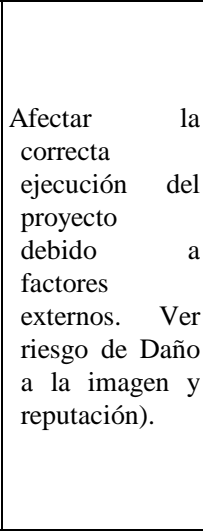 \\
\hline
\end{tabular}

\begin{tabular}{|c|c|c|c|}
\hline Externo & \begin{tabular}{|l} 
Vinculación \\
con medio \\
externo \\
afectado por \\
reforma \\
educacional, \\
limitan las \\
acciones del \\
equipo de \\
trabajo. \\
\end{tabular} & $\begin{array}{l}\text { Solicitar un informe en } \\
\text { derecho de los } \\
\text { cambios que generaría } \\
\text { la reforma educacional } \\
\text { en la relación del } \\
\text { Estado con cada } \\
\text { Universidad y cómo } \\
\text { afectaría el desarrollo } \\
\text { del Proyecto } 2030\end{array}$ & $\begin{array}{l}\text { Atrasos en el } \\
\text { desarrollo del } \\
\text { Proyecto } 2030 .\end{array}$ \\
\hline Externo & $\begin{array}{l}\text { Segmento de } \\
\text { interés } \\
\text { definido no } \\
\text { está } \\
\text { interesado en } \\
\text { las } \\
\text { actividades } \\
\text { desarrolladas } \\
\text { por er } \\
\text { Proyecto. }\end{array}$ & $\begin{array}{lrr}\text { Desde el inicio del } & \text { de } \\
\text { proyecto contar } & \text { con } \\
\text { feedback } & & \text { de } \\
\text { stakeholders } & \text { para } \\
\text { desarrollar programas } \\
\text { alineados a a las } \\
\text { necesidades. } & & \end{array}$ & $\begin{array}{l}\text { No lograr la } \\
\text { participación } \\
\text { adecuada de los } \\
\text { stakeholders. }\end{array}$ \\
\hline
\end{tabular}

Tabla $N^{\circ}$ 3: Riesgos Altos asociados a la MacroFacultad Fuente: Elaboración Propia

\section{CONSIDERACIONES ESTRATÉGICAS PARA LA MACROFACULTAD}

Conscientes de la importancia que tiene el mantener un control permanente de los posibles riesgos que puedan ocurrir e impactar en el logro de los objetivos del proyecto, es que se ha considerado el Análisis de Riesgo dentro del ámbito de Gobernanza del proyecto, reflejado en los siguientes objetivos:

- Objetivo estratégico: Propender a una gestión del cambio efectiva que favorezca la gobernanza y la sostenibilidad del proyecto.

- Objetivo específico: Implementar sistemas de gestión integral que permitan la articulación interinstitucional y la toma de decisiones efectiva.

- Actividades: Mecanismos de planificación y control para seguimiento, control de riesgos y retroalimentación del proyecto.

Para que estos objetivos sean alcanzados, se han definido las siguientes acciones específicas en este ámbito:

a. Definición de indicadores de seguimiento y monitoreo del proyecto.

b. Formalización de proceso de gestión de riesgos y estructura general responsable de su administración.

c. Medidas de mitigación y planes de contingencia asociados a la Matriz de Riesgos.

d. Actualización permanente de estado de riesgos del proyecto y acciones de mitigación implementadas.

e. Diseño lógico del sistema de control basado en un Cuadro de Control Estratégico.

f. Diseño de Reportes de Control requeridos para el Control del Proyecto.

g. Definición de Procedimientos para el ajuste de desviaciones.

Como se puede desprender del análisis anterior, existen definidas acciones que permiten tanto registrar la información asociada a los indicadores claves de riesgos, KRIs, como 
también a gestionar las medidas de mitigación en caso de ocurrencia de los riesgos. Se tiene claro además, que este análisis, debe ser actualizado durante la existencia de la MacroFacultad, de manera de ir adaptándose a nuevas fuente de generación de posibles riesgos, tanto internos como externos.

\section{CONCLUSIONES}

Es importante considerar que todo el trabajo realizado para la elaboración de la Matriz de Riesgos requiere que se realice un análisis bastante realista para definir los tratamientos que se deben considerar en cada uno de los riesgos, para evitar así que éstos se presenten, lo que implica además definir medidas de mitigaciones realistas y ejecutables.

Como se mencionó anteriormente, la formulación y planteamiento de todos los elementos de un SGR, funcionará como un mapa para la organización, como ruta de navegación que ésta debe seguir según los escenarios que se presenten. Este mapa será lo suficientemente completo dependiendo de lo completo que sea el análisis de riesgos que se realizó, por tanto, se debe cuidar tener el mayor detalle y a la vez claridad en los elementos que se postulen, considerando que a partir de lo definido previamente, se definirán los planes de acción que se llevarán a cabo.

Respecto a esto último, las acciones que se emprenderán dependerán en su totalidad de la disposición al riesgo que tenga la organización. Para la MacroFacultad, al ser un proyecto que viene regido por condiciones iniciales por parte de CORFO, donde se exigen ciertos resultados y cumplimientos de metas en plazos establecidos, por tanto existen ciertos riesgos que se deben tener en cuenta especialmente, para que no excedan en cierto rango en sus KRI definidos. Si este análisis se plantea para otro tipo de organización, que por ejemplo, esté dispuesta y pueda asumir mayores riesgos, entonces los rangos definidos para los indicadores pueden ser mayores.

En general en el Análisis de Riesgos, el plan de acción para el control de los riesgos no tiene por qué ser necesariamente la mitigación, puesto que la organización tiene total libertad de como plantea su SGR, según sus propias necesidades, objetivos y logros esperados, ya que es un sistema que se adapta a las organizaciones, y de plantearse de una forma adecuada, completa y a la vez concisa, puede transformarse en la columna vertebral de la gestión de la entidad, y debido a esta adaptabilidad, un SGR guiará a la organización hacia donde ella lo decida, otorgando la sostenibilidad que toda entidad busca (Aburto, V., \& Zapata, C. (2015)).

Una consideración muy relevante, es que una vez planteado todo el SGR, éste debe ser capaz de implementarse de forma correcta, para lo cual es necesario definir los siguientes elementos:

- Encargado del SGR

- Responsables de la medición de los KRI y herramientas que permitan recopilar la información
- Se debe construir un mecanismo de control que permita el monitoreo de los respectivos KRI, este dará la alerta al encargado cuando uno de los indicadores no esté dentro de los márgenes aceptables, los cuales serán previamente definidos por los directivos.

- Por último, cuando las alertas del monitoreo se activen, el encargado debe poder identificar el motivo del desbalance y que acciones de mitigación o correctivas deben adoptarse.

La implementación de un Sistema de Gestión de Riesgo conlleva un trabajo riguroso en todos los niveles de la organización, no solo a nivel de Dirección. Se debe considerar que hay eventos que suceden en el día a día y que no alcanzan a evidenciar los directivos, y por tanto deben considerarse todos los niveles involucrados en las operaciones de la entidad. Incorporar un SGR tendrá una dedicación de tiempo importante, en especial en la formulación y planteamiento, pero otorgará una mirada completa a la organización, y se tendrá claro hacia dónde ir en cada escenario futuro posible, es decir, es una gestión que permite la proyección sólida en el tiempo.

\section{REFERENCES}

[1] Aburto, V., \& Zapata, C. (2015). Trabajo de Título: Propuesta de un Sistema de monitoreo y reporte para los procesos de gestión de riesgo de Isapre Masvida S.A. Concepción.

[2] Amendola, J. L. (2007). Mantenimiento, Ingeniería Industrial y de Edificios. Recuperado el Agosto de 2014, de http://dialnet.unirioja.es/servlet/articulo?codigo=2263139

[3] Committee of Sponsoring Organizations of the Treadway Commission. (2004). Gestión de riesgos corporativos: marco integrado.

[4] García, M. (2007). Gestión de Riesgos de Políticas Públicas: Un gran ausente en la Reforma del Estado Perú. Recuperado el Agosto de 2014, de Gestión Pública de Perú: http://www.gestionpublica.org.pe/plantilla/rxv5t4/1029474941/en14ce/20 07/novi/revges_60.pdf

[5] González, E. J. (2013). Boletín "COSO ERM versus ISO 31000". Recuperado el Agosto de 2014, de http://www.megaprocesos.com.gt/sites/default/files/boletin_3.pdf

[6] International Organization for Standardization. (18 de noviembre de 2009). Sitio web ISO. Recuperado el 22 de Agosto de 2014, de http://www.iso.org/iso/news.htm?refid=Ref1266

[7] Roisenzvit, A., \& Zárate, M. (2006). Hacia una cultura del Risk Management: El próximo desafío para la región y cómo esto afecta los procesos de evaluación FSAP. Recuperado el Agosto de 2014, de Riesgo Operacional: http://www.riesgooperacional.com/docs/paper_alfredo.pdf 